\title{
Music intervention efficacy in elderly: a promising non-pharmacological approach to cognitive dysfunctions
}

\author{
Claudia Vinciguerra ${ }^{1}$
}

Published online: 5 June 2017

(C) Springer-Verlag Italia 2017

Aging, due to the gradual elongation of average life expectancy, represents the strongest known risk factor for cognitive decline and behavior disorders. A slow weakening of some cognitive functions such as memory, processing information speed, language, visual learning, problems solving ability, and executive functioning, with sparing of crystallized intelligence (i.e., the experience), is fairly common. These dysfunctions are often associated with changes in behavior, reduced environmental adaptation skills, depression and anxiety, which lead to a worsening of the quality of life, accounting a considerable burden due to the negative impact on various functions, including social and daily living activities $[1,2]$.

Currently, music intervention (MI) plays an important role among the drug-free treatment and rehabilitation of patients with acute and chronic neurological and somatic diseases. Also, previous studies showed its effectiveness in cognitive, mood, and behavior disorders [3].

Moreover, being non-invasive, free of adverse events and not requiring an expensive training, it can be delivered easily and successfully. The power of sound and music has been recognized in all cultures and their therapeutic use has gone through the centuries, social and political travails, cultural movements, and scientific discovery without ever being questioned, representing a part of our heritage, genetic and experience, worldwide shared since the prenatal age.

Generally, MI is focused on the relationship between body language and sound, such as interaction between perception and action, but above all it represents a form of a communication

Claudia Vinciguerra

claudiavinci@hotmail.it

1 Department of Medicine, Surgery and Neurosciences, Medical School, University of Siena, Viale Bracci 2, 53100 Siena, Italy alternative to the verbal one. It also excites emotions by taking into account some sound parameters (height, intensity, duration, and timbre) and others of dynamic type (mode, genre, executive style). A wide spectrum of MI programs (interactive or passive) are known: singing songs of the repertoire of light and popular music, music/movement association (from physical relaxation to free gestures or structured in rhythmic sequences dancing and dancing popular), instrumental improvisation, and listening music tracks $[3,4]$.

The goal is not to acquire musical skills, but to use the language of sound to open alternative communication channels.

In the elderly, MI could be very important to protect brain and potentiate the normal cognitive reserve also after different pathological processes. As Claude Levi Strauss said: "Music is a machine to suppress time."

Research in this field can count on some decades of experience and has considerably developed in relation to the demands of the medical and psychological backgrounds to have greater control and reliability in regards of the methods and the evaluation of results.

Music can induce, at the same time, different emotions and this is probably linked to the activation of different brain areas based on the perceived sound stimulus. A recent functional magnetic resonance imaging (fMRI) study showed that popular music can arouse pleasure experience and strong emotional response, probably related to characteristic patterns of brain activity [5]. Another group of researchers using binaural beats phenomenon (that occurs within the cortex when two different frequencies are presented separately to each ear) during a visuospatial working memory task demonstrated an increased response accuracy, but also modified strengths of the cortical networks during the task [6]. Moreover, many others fMRI studies conducted during music interventions, especially listening, in stroke patients reported connectivity changes in different brain networks $[7,8]$. In a recent article, Bing Xu et al. 
performed a comprehensive meta-analysis and metaregression of all available clinical trials (a total of 10 studies, 14 analyses, 966 subjects) evaluating the effects of different type of music intervention on elderly (aged 65 or over), divided in two groups: from hospital and nursing homes. The predefined primary outcome was cognitive functions (in mostly studies, cognitive function was evaluated by the MiniMental State Examination, MMSE), the secondary included behavior, depressive score, anxiety score, and quality of life, suggesting a positive effect of MI. The outcomes measured before and after a short music intervention period (variable from a few hours to a few weeks), such as the follow-up data, were extracted and analyzed by the authors [9].

The authors concluded that MI in both groups, compared to controls, plays a positive effects on cognitive functions as well as disruptive behavior, depression, anxiety, and quality of life in elderly and can be used to increase cognitively stimulating leisure activities (i.e., reading, playing chess, or playing music). These data are promising suggesting, as recommended by many health institutions, to consider the use of this nonpharmacological approach complementary to the first line treatment of cognitive dysfunctions.

A growing scientific interest in MI has been developed during the years with many new future researches. Collaboration with other disciplines and professional figures such as psychology, psychology of art, music, psychotherapists, doctors, and musicians is mandatory. Consideration should also be given to validation of methods in order to identify global strategies and techniques to apply for each clinical setting. The evaluation must be related to a methodological process that involves targets linking, variables measured, data collection, tools used, and interpretation, markedly verifying methods, and techniques performed. Moreover, an accurate standardization of music exercises performed will be needed.

In consequences, the musicologist should perform a research activity using the principles of verification and control of the methodologies applied and need to have a scientific approach, adopting innovative choices in relation to patients and the operating techniques used, identifying the specific contribution of music therapy beyond simple effects related to sound exposure.
Also, further studies need to be addressed, including more large sample sizes stratified by a single cognitive deficit through a specific neuropsychological assessment. Many efforts will be needed, in order to identify new intervention strategies and to implement a rehabilitation programs tailored to the needs, predispositions, and preferences of each patient. Also, it would be very interesting to investigate changes in brain connectivity through further fMRI studies, before and after musical interventions, taking into account the role of neuroplasticity and reserve (brain and cognitive). These strategies could be applied in the early stages of different neurological disease in order to slow down and control the degenerative process, providing a strong evidence for MI clinical utility.

\section{References}

1. Alexopoulos GS (2003) Clinical and biological interactions in affective and cognitive geriatric syndromes. Am J Psychiatry 160:811-814

2. Hornby-Turner YC, Peel NM, Hubbard RE (2017) Health assets in older age: a systematic review. BMJ Open 7(5)

3. Hagen B, Armstrong-Esther C, Sandilands M (2003) On a happier note: validation of musical exercise for older persons in long term care settings. Int J Nurs Stud 40(4):347-357

4. McCaffrey T, Edwards J (2016) Music therapy helped me get back doing: perspectives of music therapy participants in mental health services. J Music Ther 53(2):121-148

5. Chen Q, Zhang Y, Hou H et al (2017) Neural correlates of the popular music phenomenon: evidence from functional MRI and PET imaging. Eur J Nucl Med Mol Imaging 44(6):1033-1041

6. Beauchene C, Abaid N, Moran R et al (2016) The effect of binaural beats on visuospatial working memory and cortical connectivity. PLoS One 11(11):e0166630

7. Karmonik C, Brandt A, Anderson J et al (2016) Music listening modulates functional connectivity and information flow in the human brain. Brain Connect. doi:10.1089/brain.2016.0428

8. Ripollés P, Rojo N, Grau-Sánchez J et al (2016) Music supported therapy promotes motor plasticity in individuals with chronic stroke. Brain Imaging Behav 10(4):1289-1307

9. Xu B, Sui Y, Zhu C et al (2017) Music intervention on cognitive dysfunction in healthy older adults: a systematic review and metaanalysis. Neurol Sci. doi:10.1007/s10072-017-2878-9 